\title{
The Advantages of Three Dimensional Techniques (3D) in Pituitary Adenoma Treatment
}

\author{
Diabate Adama ${ }^{1}$, MEM Gar-elnabi $^{2}$, and Mohammed, A. AliOmer ${ }^{2,3}$ \\ ${ }^{1}$ University of Hail, College of Applied Medical Science-Hail, KSA \\ ${ }^{2}$ Sudan University of Science \& Technology, College of Medical Radiological Science, Khartoum - Sudan \\ ${ }^{2,3}$ Qassim University, College of Applied Medical Science, - Buraida, KSA
}

\begin{abstract}
The background of this study is to evaluate $3 D$ dose distribution in organs at risk (OARs). The sample of the study consists of 300 patients; with age-range mean and standard deviation $(35 \pm 2.32$ respectively). The CT simulator was used to collect data to delineate pituitary adenoma including growth target volume (GTV) and planning target volume (PTV 95\%) as an isodose line for treatment protocol in order to obtain dose distribution of $3 D$ dose coverage to the target while sparing organs at risks. The main results of this study were, $3 D$ dose distribution is safe, because it confines the dose to gross target volume only, while sparing escalating dose to the organ at risks (OARs), the average dose reaching the organs at risks during the pituitary adenoma to the adjacent critical organs are such as: in temporal lobe is $1.14 \mathrm{~Gy}$, in eye is $0.86 \mathrm{~Gy}$, and in optic chiasm is 0.95 Gy. Therefore, it is concluded that the $3 D$ dose distribution in pituitary adenoma treatment reduces an unnecessary radiation to critical organs. Thus, new technique needs to be developed to halt an unnecessary dose reaching organs at risks during treatment.
\end{abstract}

Keywords: radiation therapy $3 D$ technique, pituitary adenoma, organ at risks, gross tumour target, planning target volume

\section{Introduction}

The pituitary gland is the maestro gland of the body system and it controls the activities of other glands in the body, situated in sella turcica, and it is related to the critical organs of the brain such as: optic chiasma, temporal lobe, and eye. This paper will evaluate the advantages of three dimensional techniques in pituitary adenoma treatment. The three dimensions (3D) technique represents length, width, and height, any three directions are not all lie in the same plane $\left({ }^{1)}\right.$.The main objective of this study; is to provide highest probability of cure with the least radiation to normal tissues. However, three-dimensional 3D planning allows for accurate dose calculations to irregular shapes ${ }^{(2)}$.

In a retrospective study, three dimension (3D) techniques improve patient localization methods in two ways: (1) increase tumor local control and (2) diminishes in side effects and toxicities ${ }^{(3)}$. Moreover, the improvement in tumor control lies within the ability to increase the tumor dose, either by more accurately delivering $100 \%$ of the dose to the tumor or through dose-escalation ${ }^{(4)}$. On the other hand, the entire content of the sella and cavernous sinus are included in the CTV. Thus, a CTV expansion of $0.5 \mathrm{~cm}$, i.e., 1.0 to $1.5-\mathrm{cm}$ margin to determine the PTV; the margins of $7 \mathrm{~mm}$ can provide dose distribution with minimal dose to surrounding tissues (the eyes, optic nerves, optic chiasm, and temporal lobes) ${ }^{(5)}$. The concept of the planning organ at risk volume (PRV), in which a margin is added around the organ to compensate for that organ's spatial uncertainties. Therefore, 3Dtechnique increases demands on the radiation oncology physicist to insure adequate quality assurance measures for precision in tumor imaging, patient setup reproducibility, organ motion assessment, and treatment-delivery verification. This study will highlight the features of some of the common sites and how new technologies are likely to be used in the future over radiotherapy centers ${ }^{(6)}$.

\section{Methods And Materials}

The CT simulator was used to radiograph patients of pituitary adenoma after properly positioning on an immobilization device for 3D plans inside the tumour structure to delineate the gross target volume (GTV). The size of sample population is about 300 patients of pituitary adenoma, because the planning target volume (PTV) to organs at risk (OAR) limits the ability of three-dimensional 3D application to deliver a prescribed dose to PTV while sparing OAR ${ }^{(7)}$. The target volume is covered by the $95 \%$ isodose; to obtain possible minimum doses to critical organs without compromising the PTV coverage of at least $95 \%$ dose to $95 \%$ of PTV volume ${ }^{(8)}$.The Conventional CRT is used to give the total dose of 45 to 50 Gy at 1.8 Gy per fractionation ${ }^{(9)}$.

The irradiation of treatment area control remains a significant problem in management of pituitary tumour, but the dose distribution of 3D technique in pituitary tumour will eventually provide an answer to the problem in management of pituitary tumour with the ability to deliver a conformed radiation to the target volume. Thus, the height of the pituitary gland in women increased again in the 50- to 59-year-old age group ${ }^{(10)}$. 
The study population is 300 patients of pituitary adenoma whom were randomly selected from the age-groups from 10 to 57 years old according to their availability.

\section{Results}

The previous studies focused on the dose limit in pituitary adenoma treatment, regardless of tissue tolerance dose limit in general; but this study had found that, 3D dose distribution utilities in treatment of patients of pituitary adenoma while reducing dose to a surrounding tissue such as: temporal lobe, eye, optic chiasm.

\section{III.1 Temporal Lobe}

The temporal lobe presented in the fig 3.1 (a bar or histogram) shows the frequency distribution of the pituitary adenoma based on dose received by temporal lobe in 3D dose distribution. The dose received by temporal lobe in 3D dose distribution. However, the dose received by a critical organs from a tumour dose are as follow (21patients, 7\%, 0.2Gy), (43 patients, 14.3\%, 0.22 Gy), (86 patients, 28.6\%, 0.23 Gy), (108 patients, $36 \%, 0.24 \mathrm{~Gy}$ ), and (42 patients, 14\%, $0.25 \mathrm{~Gy}$ ). In addition to that, mean and standard deviation of dose received by temporal lobe in $3 \mathrm{D}$ dose distribution is $(0.23 \pm 0.013)$. Moreover, three dimension (3D) maximized dose to the temporal lobes and reduces $5 \%$ of the prescribe dose ${ }^{(11)}$. Despite of the risk of developing a clinically significant radiation optic neuropathy was with the dose receiving about 12 Gy or less. Temporal lobe necrosis is the most late-stage complication after radiation therapy ${ }^{(12)}$.

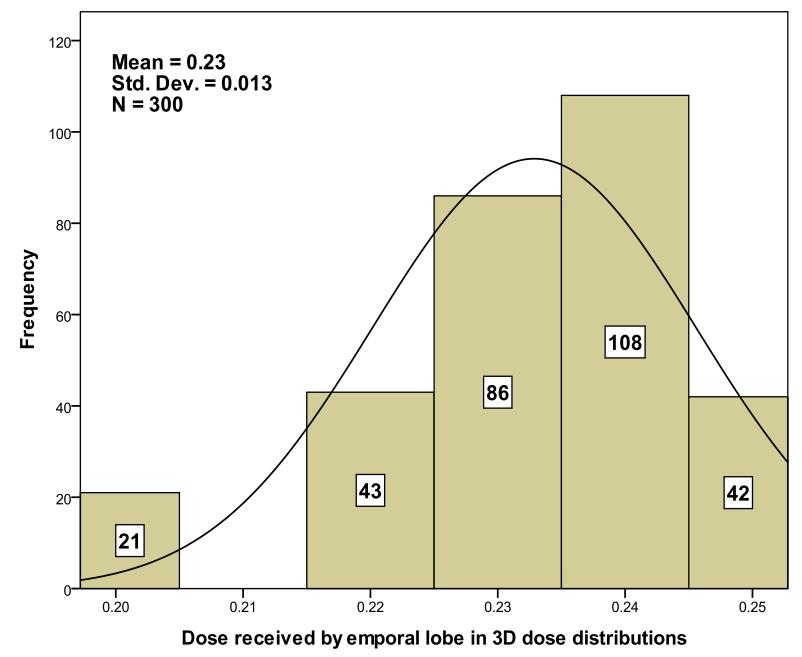

Figure 3.1 shows the study sample of temporal lobe based on dose received during 3D dose distribution in pituitary adenoma treatment.

\section{III.2 Eye}

The eye in the fig 3.2 (a bar or histogram) shows the frequency distribution of the pituitary adenoma based on dose received by eye in $3 \mathrm{D}$ dose distribution. The vertical axis shows the frequency of the dose received by eye in 3D dose distribution, the horizontal axis compares the dose level received by eye listed, divided into a comparison of four doses in Gray in 3D dose distribution in a critical organs. As it is shown in the figure 4.2 that the majority of study sample $(107$ patients, $35.6 \%$,) their eye received about $(0.23 \mathrm{~Gy})$ during the treatment of pituitary adenoma; (172 patients, 57\%) received unnecessary dose in the eye is about $(0.21-0.22$ Gy); and (21 patients, 7\%) received dose in eye is about (0.20 Gy). Thus, the mean and standard deviation of dose received by eye in $3 \mathrm{D}$ dose distribution during treatment pituitary adenoma is $(0.22 \pm 0.10)$. Nevertheless, vision can be lost in an unnecessary dose beyond $95 \%$ that will eventually cause retinal hemorrhage, this is considered to an extremely high rate of complications, and the tolerance dose to the optic nerve or chiasm is $8 \mathrm{~Gy}$ from prescribed dose ${ }^{(13)}$. 


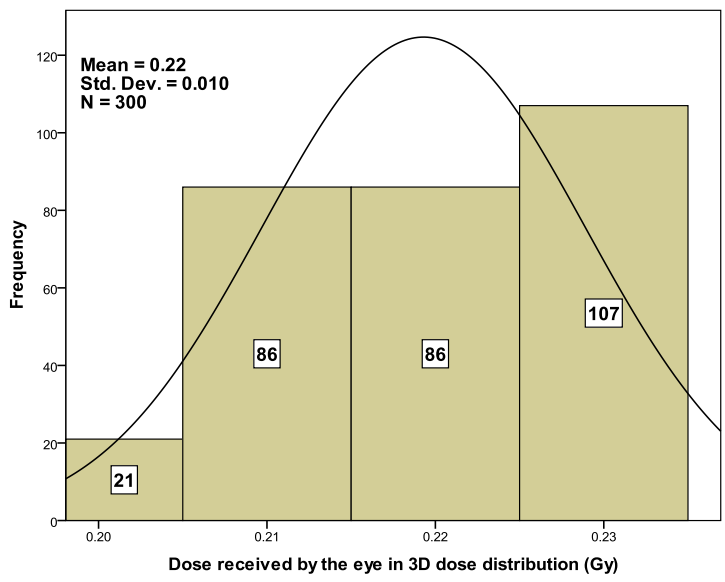

Figure 3.2 (a bar or histogram) shows the frequency distribution of the pituitary adenoma based on dose received by eye in $3 \mathrm{D}$ dose distribution.

\section{III.3 Optic chiasm}

The optic chiasma in the fig 3.3 (a bar or histogram) shows the frequency distribution of the pituitary adenoma based on dose received by optic chiasma in 3D dose distribution. The vertical axis shows the frequency of study sample, the horizontal axis compares dose received by optic chiasma in 3D dose distributions in Gy listed, divided into a comparison of six unnecessary dose reaching the optic chiasma during treatment of pituitary adenoma as follow ( $0.17 \mathrm{~Gy}, 0.18 \mathrm{~Gy}, 0.19 \mathrm{~Gy}, 0.20 \mathrm{~Gy}, 0.21 \mathrm{~Gy}$, and $0.22 \mathrm{~Gy})$. It can be seen from the figure 4.3 that the doses escalating to an optic chiasma of doses are as follow ( 92 patients, $27.7 \%, 0.17 \mathrm{~Gy}),(20$ patients, 6.6\%, $0.19 \mathrm{~Gy})$, (84 patients, 28\%, $21 \mathrm{~Gy})$, and (104 patients, 34.6\%, 0.22 Gy); but the mean and the standard deviation of the dose received by optic chiasma in 3D dose distribution is $(0.20 \pm 0.019)$. However, 3D dose distribution plans could spare optic chiasm from unnecessary radiation during treatment ${ }^{(14)}$. In addition, the optic chiasma seems to be more tolerant of radiation ${ }^{(15)}$.

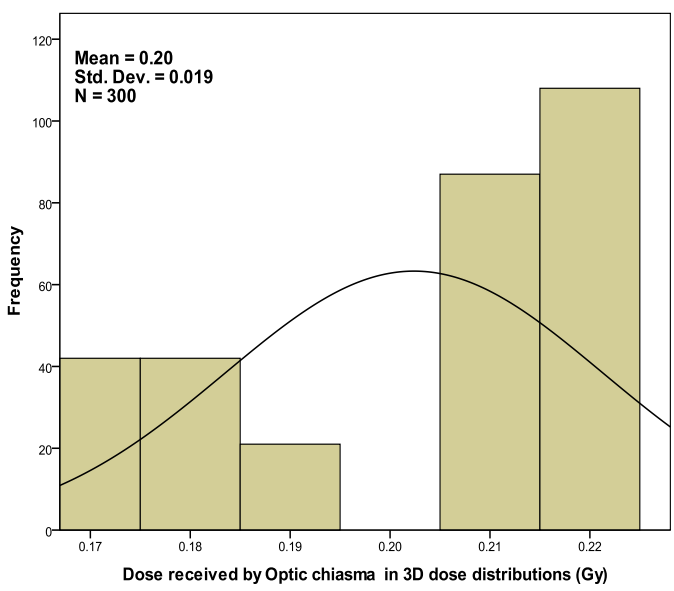

Figure 3.3 (a bar or histogram) shows the frequency distribution of the pituitary adenoma based on dose received by optic chiasm in $3 \mathrm{D}$ dose distribution.

\section{Conclusions}

In this present study, it is concluded that, the 3D dose distribution is safe and reduces the unnecessary dose reaching beyond the area of the field during the treatment; but there is no justification when radiation energy is being absorbed in the body that will produce a free radicals if the dose is less in accordance. However, these findings can be implicated in clinical applications because it lessens the unnecessary dose to the adjacent tissues. Furthermore, the limitation of the study did not find the length of these complications to be occurred and also the production enzyme repair which will fight in order to repair damage caused by the minor unnecessary radiation embedded in the tissues; new method should be used to treat pituitary adenoma cases so that to conform the dose to the bed of tumour need to be developed for further scientific researches.. 


\section{Acknowledgments}

This work is dedicated to the people who are persevering in the fight of their lives against pituitary adenoma. When the doctors say "it's over" that doesn't always mean it's over! For any patient who is determined to continue the fight against cancer, we are committed to providing every reasonable option of treatment technique available, with the best level of precision, skill and knowledge humanly possible during the treatment of pituitary adenoma patients.

\section{References}

[1]. Dale Rolfsen, Three-dimensional space. Accessed on 5 /10/2013. Available from http://en.wikipedia .org/wiki/Threedimensional_space\#References.

[2]. Kara Bucci, Alison Bevan, Mack Roach, M, Advances in radiation therapy: conventional to 3d, to IMRT, to 4d, and beyond, Cancer J Clin; 55(117), 2005, 134.

[3]. Bucci M, Xia P, Lee N., Intensity modulated radiation therapy for carcinoma of the nasopharynx: an update of the UCSF experience, Int J Radiat Oncol Biol Phys, 60, 2004, 317-318.

[4]. Cooper JS, Pajak TF, Forastiere AA., Postoperative concurrent radiotherapy and chemotherapy for high-risk squamous-cell carcinoma of the head and neck, N Engl J Med; 350, 2004, 1937-1944.

[5]. Solit DB, Goldwein JW., Posterior fossa: analysis of a popular technique for estimating the location in children with medulloblastoma. Radiology 195, 1995, 697-698.

[6]. International Commission of Radiation Units and Measurements, ICRU, Prescribing, Recording and Reporting Photon Beam Therapy, report no 50, 1993. Bethesda.

[7]. Jillian Maclean, Christopher Stacey, Naomi Fersht, Ivan Rosenberg, Derek D’Souza, Julia Solano, Nazima Haji and Susan Short, "Arc Delivered Intensity-Modulated Radiotherapy Achieves Highly Conformal Radiotherapy for Brain Tumor Patients with Minimal Hair Loss, EJCMO, 3( 4) ,2011, 22-24.

[8]. Hitchen CJ, Osa EO, Dewyngaert JK, Chang J, Narayana A., Dose to craniofacial region through portal imaging of pediatric brain tumors, J Appl Clin Med Phys, 13(1),2012, 33-85.

[9]. Vassilis Kouloulias, Stella Thalassinou, Kalliopi Platoni, Anna Zygogianni, John Kouvaris, Christos Antypas, Efstathios Efstathopoulos, Kelekis Nikolaos, (2013), 'The Treatment Outcome and Radiation-Induced Toxicity for Patients with Head and Neck Carcinoma in the IMRT Era: A Systematic Review with Dosimetric and Clinical Parameters', BioMed Research International Vol. 2013 , Article ID 401261,2013, 12

[10]. Tsunoda. A., O. Okuda, and K. Sato, MR Height of the Pituitary Gland as a Function of Age and Sex: Especially Physiological Hypertrophy in Adolescence and in Climacterium, AJNR Am J Neuroradiol, 18, 1997, 551-554.

[11]. Chau RM, Teo PM, Choi PH, Cheung KY, Lee WY, Three-dimensional dosimetric evaluation of a conventional radiotherapy technique for treatment of nasopharyngeal carcinoma, Radiother Oncol, 58( 2),2001, 143-53

[12]. Stafford SL, Pollock BE, Leavitt JA, Foote RL, Brown PD, Link MJ,Gorman DA, Schomberg PJ., A study on the radiation tolerance of the optic nerves and chiasm after stereotactic radiosurgery, Int J Radiat Oncol Biol Phys. 55(5), $2003,1177-81$.

[13]. Robert J. Cassady, MD. Response of the Normal Eye to High Dose Radiotherapy, Oncology, June 01, 1996.

[14]. Kamian S, Kazemian A, Esfahani M, Mohammadi E, Aghili M.,Comparison of three-dimensional vs. conventional radiotherapy in saving optic tract in paranasal sinus tumors, J BUON, 15(2),2010, 281-4.

[15]. Hasegawa T, Kobayashi T, Kida, Y., Tolerance of the optic apparatus in single-fraction irradiation using stereotactic radiosurgery: evaluation in 100 patients with craniopharyngioma, Neurosurgery, 66(4), 2010, 688-94. 